\title{
ABOUT THE DECISION OF REACHABILITY FOR REGISTER MACHINES
}

\author{
VÉRONIQUe CORTIER ${ }^{1}$
}

\begin{abstract}
We study the decidability of the following problem: given $p$ affine functions $f_{1}, \ldots, f_{p}$ over $\mathbb{N}^{k}$ and two vectors $v_{1}, v_{2} \in \mathbb{N}^{k}$, is $v_{2}$ reachable from $v_{1}$ by successive iterations of $f_{1}, \ldots, f_{p}$ (in this given order)? We show that this question is decidable for $p=1,2$ and undecidable for some fixed $p$.
\end{abstract}

Mathematics Subject Classification. 68Q60.

\section{INTRODUCTION}

Reachability is a fundamental question for computation models: a typical safety property of a reactive system is the unreachability of some catastrophic state. Reachability is straightforwardly decidable (in a time linear in the number of states) for finite-state systems. For other (infinite-state) computation models, it is most of the time undecidable.

In this paper, we study the border between decidability and undecidability for a particular computation model: configurations are vectors of non-negative integers. Each move from a configuration to its successor is given by an affine function $f(X)=A X+B$ where $A$ is a matrix of non-negative integers and $B$ is a vector of integers. Such affine functions are used to model the evolution of dynamical systems like the age repartition of trees of a forestry development or the human population growth (see [9]): the initial vector represents the initial repartition and the affine function describe the evolution of this repartition during a year. They can also be used to compute limit trajectories (see [1]).

Keywords and phrases: Verification, infinite state systems.

1 Laboratoire Spécification et Vérification, École Normale Supérieure de Cachan, CNRS, 61 avenue du Président Wilson, 94230 Cachan, France; e-mail: cortier@lsv.ens-cachan.fr (C) EDP Sciences 2003 
Petri nets with transfer are a particular case of this model (components of $A$ are 0 or 1 ), hence reachability is in general undecidable, see [6]. Many register machines can be also modeled using such a computation model.

On the decidability side, in [2] B. Boigelot shows that

$$
\left\{f_{1}^{k_{1}} \cdots f_{p}^{k_{p}}(X) \mid X \in \mathbb{N}^{m}, k_{1}, \ldots, k_{p} \in \mathbb{N}\right\}
$$

is definable in $W S_{1} S$ (weak monadic second order logic with one successor), hence reachability is decidable, when $f_{1}, \ldots, f_{k}$ are affine functions such that the matrix $A_{1}, \ldots, A_{n}$ are diagonalizing and their eigenvalues satisfy some conditions.

Instead of restricting the operations on the vectors, we consider here some restriction on the control. For instance, it has been shown in [3] that reachability for extended counter machines becomes decidable when the control is flat. We consider here the iteration of some affine functions with such a flat control. More precisely, given arbitrary affine functions $f_{1}, \ldots, f_{p}$, we assume that $f_{1}, \ldots, f_{p}$ are applied in a fixed order: first $f_{1}$ is applied an arbitrary number of times and then $f_{1}$ is not used again, then $f_{2}$ is applied an arbitrary number of times and then $f_{2}$ is not used again, etc. Under these conditions, we prove that reachability is decidable for $p=1$ (Sect. 2.1), for $p=2$ (Sect. 3) and undecidable for some $p$ (Sect. 4).

\section{Preliminaries}

\subsection{INTRODUCTION}

Notation: $\mathcal{A}_{k}(\mathbb{N})$ is the set of affine functions $f: \mathbb{N}^{k} \rightarrow \mathbb{N}^{k}$ such that $f(X)=$ $A X+B$ where $A$ is a matrix with nonnegative integer components and $B$ is a vector in $\mathbb{Z}^{k}$.

Notation: $M_{k}(\mathbb{N})$ is the set of matrices of size $k \times k$ with nonnegative integer components.

Notation: $E_{i}^{k}$ denotes the vector in $\mathbb{N}^{k}$ such that the $j^{\text {th }}$ coordinate is 1 if $j=i$, 0 otherwise.

We consider the following decision problem: given $f_{1}, \ldots, f_{p} \in \mathcal{A}_{k}(\mathbb{N})$, given $U, V \in \mathbb{N}^{k}$, does $V$ belong to $\left\{f_{p}^{n_{p}} \cdots f_{1}^{n_{1}}(U) \mid n_{1}, \ldots, n_{p} \in \mathbb{N}\right\}$ ?

Example 1.1. We consider

$$
f(X)=\left(\begin{array}{lll}
1 & 0 & 0 \\
0 & 1 & 0 \\
0 & 1 & 1
\end{array}\right) X+\left(\begin{array}{c}
-1 \\
0 \\
0
\end{array}\right)
$$




\begin{tabular}{|c|c|}
\hline Petri net extension & affine function \\
\hline Petri net & $A=I d$ \\
Double Petri net & $I d \leq A \leq 2 I d$ \\
Generalized Transfer Petri net & $0 \leq A \quad \forall j A_{j} \neq 0$ \\
Reset Petri net & $0 \leq A \leq I d$ \\
\hline
\end{tabular}

FiguRE 1. Example of transition classes which can be modeled by $f(X)=A X+B$, cf. [7].

Then $f\left(\begin{array}{l}a \\ b \\ c\end{array}\right)=\left(\begin{array}{c}a-1 \\ b \\ c+b\end{array}\right)$ if $a \geq 1, \quad f^{n}\left(\begin{array}{l}a \\ b \\ c\end{array}\right)=\left(\begin{array}{c}a-n \\ b \\ c+n b\end{array}\right)$ if $a \geq n$. If $a=0, f\left(\begin{array}{l}a \\ b \\ c\end{array}\right)$ is not defined because $f(X)$ is not allowed to take negative values.

Then, given $U=\left(\begin{array}{l}a \\ b \\ 0\end{array}\right)$ and $V=\left(\begin{array}{l}0 \\ b \\ c\end{array}\right)$, where $a, b . c \in \mathbb{N}$, deciding if $V$ belongs to $\left\{f^{n}(U) \mid n \in \mathbb{N}\right\}$ is equivalent to decide if $c=a b$.

This type of transition functions $(f(X)=A X+B)$ is more general than many other transitions which are considered in the literature (Fig. 1). For example, if $A=I d$, we obtain a Petri net. But, on the other hand, there is a strong restriction on the control: $f_{1}, \ldots, f_{p}$ have to be iterated in a fixed order, which is not the case in Petri nets. Such a control corresponds to the notion of "flat automata" in $[3]$.

\subsection{USEFUl PROPERTIES}

We consider a partial order on vectors of integers in the following way:

Definition 1.2. Let $U, V \in \mathbb{N}^{k}, \quad U \leq V$ if and only if $U$ 's coordinates are all smaller than those of $V$.

The relation $\leq$ is a well quasi-order [5]. Moreover, it is easy to verify that for every $f \in \mathcal{A}_{k}(\mathbb{N})(f(X)=A X+B), f$ is "increasing" for $\leq$ : if $U_{1} \leq U_{2}$ then $f\left(U_{1}\right) \leq f\left(U_{2}\right)$. This last property uses that $A$ has only non-negative components but does not require anything on $B$ 's components. That is why we can allow $B$ to have negative components.

Notation: We write $V \widetilde{>} U$ if at least one of $V$ 's coordinates is greater than the corresponding one of $U$, i.e., if $U \ngtr V$.

Clearly, if $V_{1} \geq V_{2}$ and $V_{2} \widetilde{>} U$ then $V_{1} \widetilde{>} U$.

Notation: The size of $V$, written $|V|$, is the sum of the absolute values of its coordinates. 


\section{Decidability Results}

\subsection{Decidability for One FunCtion}

Since $\leq$ is a well quasi-order and affine functions are increasing for $\leq$, the problem stated in introduction is decidable when $p=1$.

Theorem 2.1. Given $f \in \mathcal{A}_{k}(\mathbb{N}), U, V \in \mathbb{N}^{k}, V \in\left\{f^{n}(U) \mid n \in \mathbb{N}\right\}$ is decidable.

Proof. We consider the following sequence: $U, f(U), f^{2}(U), \ldots, f^{n}(U), \ldots$

If there exists $N$ such that $f^{N}(U) \nsupseteq 0$, then the sequence is finite and $V \in$ $\left\{f^{n}(U) \mid n \in \mathbb{N}\right\}$ is decidable.

Otherwise there exist $N_{1}<N_{2}$ such that $f^{N_{1}}(U) \leq f^{N_{2}}(U)$ because $\leq$ is a well quasi-order. Let $l=N_{2}-N_{1}$. For all $0 \leq i \leq l-1$,

$$
f^{N_{1}}(U) \leq f^{N_{2}}(U) \Rightarrow f^{i}\left(f^{N_{1}}(U)\right) \leq f^{i}\left(f^{N_{2}}(U)\right)
$$

since $f$ is increasing, thus $f^{N_{1}+i}(U) \leq f^{N_{2}+i}(U)$.

So we have:

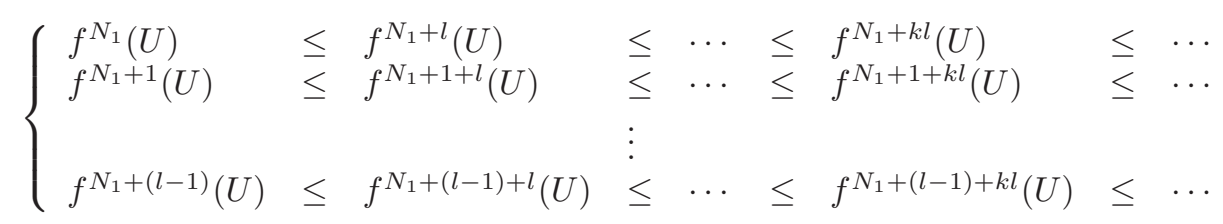

- Either for one of these sequences two consecutive terms are equal, then this sequence becomes constant.

- Or all these sequences are strictly increasing.

In concrete terms, we proceed on the following way: we compute successively $U, f(U), f^{2}(U), \ldots, f^{n}(U), \ldots$ (and at each step we check the equality to $V$ ) until we find $f^{N_{1}}(U) \leq f^{N_{2}}(U)$, unless the sequence is finite.

In this case, the algorithm stops, there is no $n$ such that $V=f^{n}(U)$.

In the other case (if we find $f^{N_{1}}(U) \leq f^{N_{2}}(U)$ ), we compute successively

$$
f^{N_{1}}(U) \leq f^{N_{1}+l}(U) \leq f^{N_{1}+2 l}(U) \leq \cdots \leq f^{N_{1}+k l}(U) \leq \cdots
$$

until either one of the coordinates is greater than the corresponding one of $V$, or the sequence becomes constant (if two consecutive terms are equal). In the second case, $\left\{f^{n}(U) \mid n \in \mathbb{N}\right\}$ is a finite set: we check if $V \in\left\{f^{n}(U) \mid n \in \mathbb{N}\right\}$. Otherwise (if one of the coordinates is greater than the corresponding one of $V$ ), we try again with

$$
f^{N_{1}+1}(U) \leq f^{N_{1}+1+l}(U) \leq f^{N_{1}+1+2 l}(U) \leq \cdots \leq f^{N_{1}+1+k l}(U) \leq \cdots
$$

and so on with the $l$ sequences. 
Nevertheless, even if the algorithm is effective, we have no bound regarding its complexity. There is another algorithm which is more complicated, yielding however an explicit upper bound which is a tower of exponentials whose height depends linearly on $k$, the number of registers, see [4]. The main idea of this algorithm is to associate to the matrix $A$ its dependence graph $G_{A}$ (there is an edge between $i$ and $j$ in $G_{A}$, labelled by $A_{i, j}$ if and only if $A_{i, j} \neq 0$ ). Then, we break down the graph $G_{A}$ into strongly connected components and study precisely the behavior of each component when the affine function is iterated.

\section{DeCidABility FOR TWO FUnCTIONS}

A result similar to Theorem 2.1 also holds for the composition of two functions in a given order.

Theorem 3.1. Given $f, g \in \mathcal{A}_{k}(\mathbb{N}), U, V \in \mathbb{N}^{k}$, then $V \in\left\{g^{n} f^{m}(U) \mid n, m \in \mathbb{N}\right\}$ is decidable.

Proof. we proceed with three steps:

1. for $m$ fixed, we compute $n_{m}$ such that either $\left(g^{n} f^{m}(U)\right)_{n \geq n_{m}}$ is not defined or $\left(g^{n} f^{m}(U)\right)_{n \geq n_{m}}$ is periodic or $\forall n \geq n_{m}, g^{n} f^{m}(U) \widetilde{>} V$;

2. for $n$ fixed, we show that $V \in\left\{g^{n} f^{m}(U) \mid m \in \mathbb{N}\right\}$ is decidable;

3 . we combine the first two steps with a kind of cross-ruling.

\subsection{BeHAVIOR OF $\left(g^{n} f^{m}(U)\right)_{n}, m$ FIXED}

We just give here a refinement of Theorem 2.1.

Theorem 3.2. Given $f, g \in \mathcal{A}_{k}(\mathbb{N}), U, V \in \mathbb{N}^{k}$, given $m \in \mathbb{N}$, there exists $n_{m} \in \mathbb{N}$ (computable) such that:

1. either $\forall n \geq n_{m} \quad g^{n} f^{m}(U) \widetilde{>} V$;

2. or there exist $N_{m}, l_{m}$ (computable) such that $\forall n \geq n_{m} \quad \forall i<l_{m} \quad g^{N_{m}+i+n l_{m}} f^{m}(U)=g^{N_{m}+i+n_{m} l_{m}} f^{m}(U)$, i.e., there exist $N_{m}^{\prime}, l_{m}$ such that $\forall n \geq N_{m}^{\prime} \quad \exists i<l_{m} \quad g^{n} f^{m}(U)=$ $g^{N_{n}^{\prime}+i} f^{m}(U)$;

3. or $g^{n_{m}} f^{m}(U) \geq 0$, thus for all $n \geq n_{m}, g^{n} f^{m}(U)$ is not defined.

Proof. We just refine the proof of Theorem 2.1:

Let $U^{\prime}=f^{m}(U)$, we consider the following sequence: $U^{\prime} f\left(U^{\prime}\right), f^{2}\left(U^{\prime}\right), \ldots$, $f^{n}\left(U^{\prime}\right), \ldots$ Either there exists $n_{m}$ such that $g^{n_{m}} f^{m}\left(U^{\prime}\right) \geq 0$ (case 3).

Or there exist $N_{1}<N_{2}$ such that $g^{N_{1}}\left(U^{\prime}\right) \leq g^{N_{2}}\left(U^{\prime}\right)$. In this case, we consider the following sequences:

$$
\left\{\begin{array}{c}
g^{N_{1}}\left(U^{\prime}\right) \leq g^{N_{1}+l}\left(U^{\prime}\right) \leq \cdots \leq g^{N_{1}+k l}\left(U^{\prime}\right) \leq \cdots \\
g^{N_{1}+1}\left(U^{\prime}\right) \leq g^{N_{1}+1+l}\left(U^{\prime}\right) \leq \cdots \leq g^{N_{1}+1+k l}\left(U^{\prime}\right) \leq \cdots \\
\vdots \\
g^{N_{1}+(l-1)}\left(U^{\prime}\right) \leq g^{N_{1}+(l-1)+l}\left(U^{\prime}\right) \leq \cdots \leq g^{N_{1}+(l-1)+k l}\left(U^{\prime}\right) \leq \cdots
\end{array}\right.
$$


- Either for one of these sequences, two consecutive terms are equal, thus this sequence is stabilized which implies that all these sequences are stabilized (case 2).

- Or all these sequences are increasing and we compute each of them until one of the coordinates of a term of the sequence is greater than the corresponding one of $V$ (case 1$)$.

\subsection{BeHAVIOR OF $\left(g^{n} f^{m}(U)\right)_{m}, n$ FIXED}

To control $\left(g^{n} f^{m}(U)\right)_{m}$, we first establish a very useful lemma.

Lemma 3.3. Let $f, g \in \mathcal{A}_{k}(\mathbb{N}), U \in \mathbb{N}^{k}$, let $l \in \mathbb{N}, l>0$. We consider the following sequence: $g^{n}(U), g^{n} f^{l}(U), g^{n} f^{2 l}(U), \ldots, g^{n} f^{m l}(U), \ldots$ (n fixed), then either the sequence is eventually stabilized or it is never constant more than $k+1$ steps.

More formally, this sequence has the following property:

$$
\begin{aligned}
\forall m_{0} \quad\left(g^{n} f^{m_{0} l}(U)=g^{n} f^{\left(m_{0}+1\right) l}(U)=\right. & \cdots=g^{n} f^{\left(m_{0}+k+1\right) l}(U) \\
& \left.\Longrightarrow \forall m \geq m_{0} \quad g^{n} f^{m l}(U)=g^{n} f^{m_{0} l}(U)\right) .
\end{aligned}
$$

Proof. The proof of this lemma uses elementary properties of algebra.

Assume $g^{n} f^{m_{0} l}(U)=g^{n} f^{\left(m_{0}+1\right) l}(U)=\cdots=g^{n} f^{\left(m_{0}+k+1\right) l}(U)$. Let us show that $g^{n} f^{\left(m_{0}+k+2\right) l}(U)=g^{n} f^{m_{0} l}(U)$, which proves by induction that

$$
\forall m \geq m_{0} \quad g^{n} f^{m l}(U)=g^{n} f^{m_{0} l}(U) .
$$

$f^{\left(m_{0}+1\right) l}(U)-f^{m_{0} l}(U), \ldots, f^{\left(m_{0}+k+1\right) l}(U)-f^{m_{0} l}(U)$ are $k+1$ vectors of the $k$ dimensional vector space $\mathbb{Q}^{k}$, so they are linearly dependent in $\mathbb{Q}$, thus they are linearly dependent in $\mathbb{Z}$ (by multiplying by an appropriate integer). Thus

$$
\exists q_{1}, \ldots, q_{k+1} \in \mathbb{Z} \quad \sum_{i=1}^{k+1} q_{i}\left(f^{\left(m_{0}+i\right) l}(U)-f^{m_{0} l}(U)\right)=0 .
$$

Let $N$ denote the greatest $i$ such that $q_{i}$ is different from $0(1 \leq N \leq k+1)$.

Then $q_{N}\left(f^{\left(m_{0}+N\right) l}(U)-f^{m_{0} l}(U)\right)+\sum_{i=1}^{N-1} q_{i}\left(f^{\left(m_{0}+i\right) l}(U)-f^{m_{0} l}(U)\right)=0$.

Applying $f^{k+2-N}$ yields:

$$
\begin{aligned}
q_{N}\left(f^{\left(m_{0}+k+2\right) l}(U)\right. & \left.-f^{\left(m_{0}+k+2-N\right) l}(U)\right) \\
& +\sum_{i=1}^{N-1} q_{i}\left(f^{\left(m_{0}+i+k+2-N\right) l}(U)-f^{\left(m_{0}+k+2-N\right) l}(U)\right)=0,
\end{aligned}
$$


so,

$$
\begin{aligned}
q_{N} g^{n}\left(f^{\left(m_{0}+k+2\right) l}(U)\right. & \left.-f^{\left(m_{0}+k+2-N\right) l}(U)\right) \\
& +\underbrace{\sum_{i=1}^{N-1} q_{i} g^{n}\left(f^{\left(m_{0}+i+k+2-N\right) l}(U)-f^{\left(m_{0}+k+2-N\right) l}(U)\right)}_{=0}=0
\end{aligned}
$$

since (by hypothesis)

$$
\forall 1 \leq i \leq N-1 \quad g^{n} f^{(m_{0}+\overbrace{i+k+2-N}^{1 \leq}) l}(U)=g^{n} f^{(m_{0}+\overbrace{k+2-N+1}^{1 \leq} \leq k+1} l(U) .
$$

Conclusion: $g^{n} f^{\left(m_{0}+k+2\right) l}(U)=g^{n} f^{m_{0} l}(U)$.

Definition 3.4. A sequence is $k$-almost increasing if this sequence is non-decreasing and if it is never constant more than $k$ steps.

Lemma 3.5. For $n$ fixed, $V \in\left\{g^{n} f^{m}(U) \mid m \in \mathbb{N}\right\}$ is decidable.

Proof. We compute the sequence $\left(f^{m}(U)\right)_{m}$ until:

either there exists $N$ such that $f^{N}(U) \nsupseteq 0$, the sequence stops and we test if $V \in\left\{g^{n} f^{m}(U) \mid m<N\right\}$

or there exist $N, l$ such that $f^{N}(U) \leq f^{N+l}(U)$, then

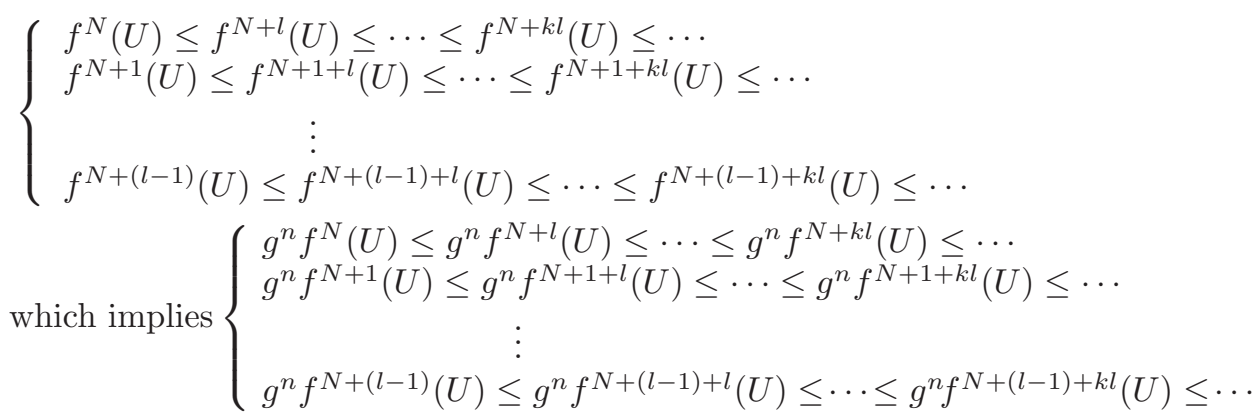

Using Lemma 3.3 (with $U^{\prime}=f^{N}(U)$ ), each sequence is:

- either $(k+1)$-almost increasing;

- or eventually stabilized.

Hence, for each of these sequences, there exists $m_{i}$ (computable) such that $\forall m \geq$ $m_{i}$ :

- either $g^{n} f^{N+i+m l}(U) \widetilde{>} V$, thus each term of the sequence $\left(g^{n} f^{N+i+m l}(U)\right)_{m \geq m_{i}}$ is different to $V$;

- or $g^{n} f^{N+i+m l}(U)=g^{n} f^{N+i+m_{i} l}(U)$.

In concrete terms, we compute each term $W$ of $\left(g^{n} f^{N_{1}+i+m l}(U)\right)_{m}$ until:

- either $W \widetilde{>} V$ which implies $V \notin\left\{g^{n} f^{m}(U) \mid m \in \mathbb{N}\right\}$; 
- or $k+2$ consecutive terms have the same value, it means that the sequence is stabilized, which implies $V \notin\left\{g^{n} f^{m}(U) \mid m \in \mathbb{N}\right\}$;

- $W=V$ which implies $V \in\left\{g^{n} f^{m}(U) \mid m \in \mathbb{N}\right\}$.

One of these 3 cases is bound to happen.

\subsection{Proof of Theorem 3.1}

We need to control $g^{n} f^{m}(U)$ when $n$ and $m$ vary at the same time. We first establish a technical lemma used to initialize the proof of Theorem 3.1.

Lemma 3.6. Let $f, g \in \mathcal{A}_{k}(\mathbb{N}), U \in \mathbb{N}^{k}$, let $N_{1}, l \in \mathbb{N}^{k}, N_{1}, l>0$.

If $f^{N_{1}}(U) \leq f^{N_{1}+l}(U) \leq f^{N_{1}+2 l}(U) \leq \cdots \leq f^{N_{1}+m l}(U) \leq \cdots$

$$
\text { and if }\left\{\begin{array}{cc}
\exists n_{0} & g^{n_{0}} f^{N_{1}}(U) \nsupseteq 0 \\
\exists n_{1} & g^{n_{1}} f^{N_{1}+l}(U) \nsupseteq 0 \\
& \vdots \\
\exists n_{k} & g^{n_{k}} f^{N_{1}+k l}(U) \nsupseteq 0
\end{array}\right.
$$

where $n_{i}$ is the smallest $n$ such that $g^{n} f^{N_{1}+i l}(U) \ngtr 0$, then

1. $n_{0} \leq n_{1} \leq \cdots \leq n_{k}$

2. $\forall m \geq k \quad g^{n_{k}} f^{N_{1}+m l}(U) \nsupseteq 0$.

Proof. The proof of (1) is easy: $g^{n_{0}-1} f^{N_{1}+l}(U) \geq g^{n_{0}-1} f^{N_{1}}(U) \geq 0$ thus $n_{1} \geq n_{0}$ and so on.

The proof of (2) uses again elementary results of algebra.

$f^{N_{1}}(U), \ldots, f^{N_{1}+k l}(U)$ are $k+1$ vectors linearly dependent in $\mathbb{Q}$ thus in $\mathbb{Z}$, thus:

$$
\exists p_{0}, \ldots, p_{k} \in \mathbb{Z} \quad \sum_{i=0}^{k} p_{i} f^{N_{1}+i l}(U)=0 .
$$

Let $N$ denote the greatest $i$ such that $p_{i}$ is not equal to 0 . Assume $p_{N}>0$ (if it is not the case, multiply the equation by -1$)$.

Let $I=\left\{i \mid p_{i}>0, i \neq N\right\}, J=\left\{j \mid p_{j}<0\right\}$. For $j \in J$, let $q_{j}=-p_{j}>0$.

$$
p_{N} f^{N_{1}+N l}(U)+\sum_{i \in I} p_{i} f^{N_{1}+i l}(U)=\sum_{j \in J} q_{j} f^{N_{1}+j l}(U)
$$

which implies

$$
p_{N} f^{N_{1}+N l}(U) \leq \sum_{j \in J} q_{j} f^{N_{1}+j l}(U)
$$

applying $f^{(k+1-N) l}$ yields:

$$
p_{N} f^{N_{1}+(k+1) l}(U) \leq \sum_{j \in J} q_{j} f^{N_{1}+\overbrace{(k+1-N+j)}^{\leq k}} l(U)
$$




$$
\Rightarrow p_{N} g^{n_{k}} f^{N_{1}+(k+1) l}(U) \leq \sum_{j \in J} q_{j} \underbrace{g^{n_{k}} f^{N_{1}+(k+1-N+j) l}(U)}_{i_{0}^{\text {th }} \text { coordonate }<0} .
$$

Applying $g^{n_{k}}$ to $f^{N_{1}}(U) \leq \cdots \leq f^{N_{1}+k l}(U)$ yields

$$
g^{n_{k}} f^{N_{1}}(U) \leq \cdots \leq g^{n_{k}} f^{N_{1}+k l}(U) .
$$

Since $g^{n_{k}} f^{N_{1}+k l}(U) \geq 0$, there exists $i_{0}$ such that the $i_{0}^{\text {th }}$ coordinate of $g^{n_{k}} f^{N_{1}+k l}(U)$ is negative, so for all $1 \leq i \leq k$, the $i_{0}^{\text {th }}$ coordinate of $g^{n_{k}} f^{N_{1}+i l}(U)$ is negative. Thus (using 2), the $i_{0}^{\text {th }}$ coordinate of $g^{n_{k}} f^{N_{1}+(k+1) l}(U)$ is negative, so:

$$
g^{n_{k}} f^{N_{1}+(k+1) l}(U) \geq 0 .
$$

Hence $\forall m \geq k \quad g^{n_{k}} f^{N_{1}+m l} \nsupseteq 0$ by induction.

We are now ready to prove Theorem 3.1.

Proof of Theorem 3.1. We consider the sequence $\left(f^{n}(U)\right)_{n}$.

- Either there exists $N$ such that $f^{N}(U) \nsupseteq 0$. It is the easy case:

$$
\left\{g^{n} f^{m}(U) \mid n, m \in \mathbb{N}\right\}=\left\{g^{n} f^{m}(U) \mid n \in \mathbb{N}, m<N\right\} .
$$

For all $m<N$, we test if $V \in\left\{g^{n} f^{m}(U) \mid n \in \mathbb{N}\right\}$.

- Or there exist $N, l$ such that $f^{N}(U) \leq f^{N+l}(U) \leq \cdots \leq f^{N+k l}(U) \leq \cdots$ Let us show that $V \in\left\{g^{n} f^{N+m l}(U) \mid n, m \in \mathbb{N}\right\}$ is decidable, which proves that $V \in\left\{g^{n} f^{N+i+m l}(U) \mid n, m \in \mathbb{N}\right\}$ is decidable for all $1 \leq i \leq l$ (take $U^{\prime}=f^{i}(U)$ ). Thus it implies $V \in\left\{g^{n} f^{m}(U) \mid n, m \in \mathbb{N}\right\}$ is decidable.

We first consider the sequence $\left(g^{n} f^{N}(U)\right)_{n}$. Either there exists $n_{0}$ such that $g^{n_{0}} f^{N}(U) \nsupseteq 0$, then we consider the sequence $\left(g^{n} f^{N+l}(U)\right)_{n}$, or there exist $n_{0}, l_{0}$ such that $g^{n_{0}} f^{N}(U) \leq g^{n_{0}+l} f^{N}(U) \leq \cdots$, thus $\forall n \quad g^{n} f^{N}(U) \geq 0$. We repeat this (at most $k$ times) until we obtain Case 1 or Case 2.

\section{Case 1.}

$$
\begin{array}{cc}
\exists n_{0} \quad g^{n_{0}} f^{N_{1}}(U) \nsupseteq 0 \\
\exists n_{1} \quad g^{n_{1}} f^{N_{1}+l}(U) \nsupseteq 0 \\
\vdots \\
\exists n_{k} \quad g^{n_{k}} f^{N_{1}+k l}(U) \nsupseteq 0 .
\end{array}
$$

Applying Lemma 3.6 yields $\forall m \geq k \quad g^{n_{k}} f^{N_{1}+m l}(U) \geq 0$. Thus, it is enough to test if $V \in\left\{g^{n} f^{N+m l}(U) \mid m \in \mathbb{N}\right\}$ for all $n<n_{k}$ (see Fig. 2).

Case 2. There exists $i \leq k$ such that $\forall n \quad g^{n} f^{N+i l}(U) \geq 0$. We rename $N:=N+i l$. So,

$$
\forall n \forall m \quad g^{n} f^{N+m l}(U) \geq g^{n} f^{N}(U) \geq 0 .
$$




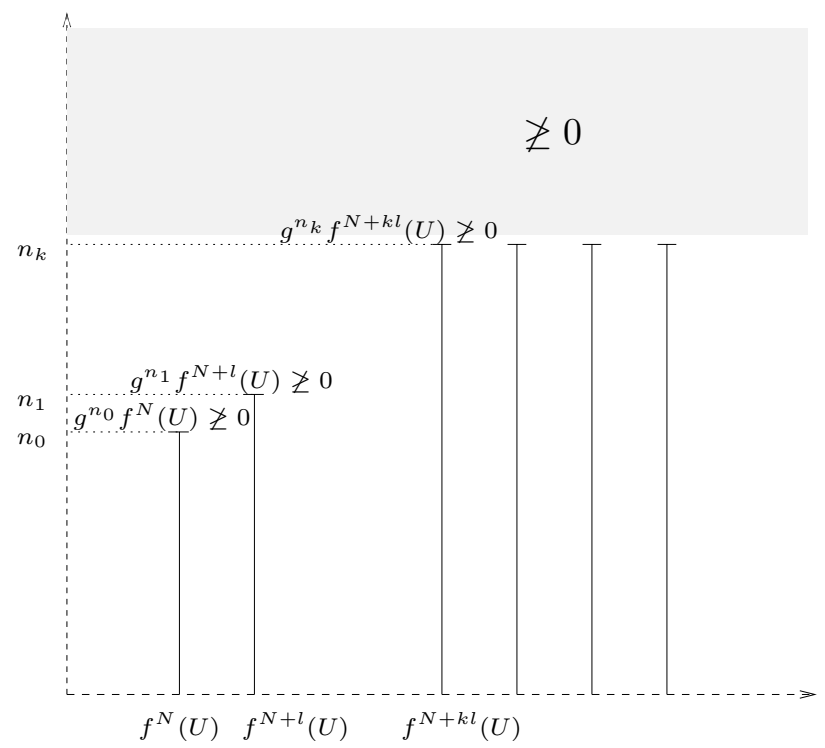

Figure 2. Case 1.

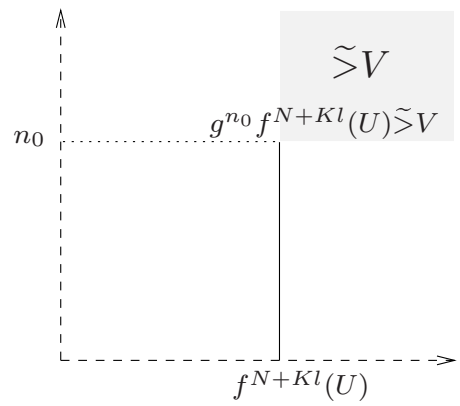

Figure 3. Case 2.

Let $K=(k+1)|V|$. We consider $\left(g^{n} f^{N+K l}(U)\right)_{n}$. Applying Theorem 3.2 yields 3 cases.

1. There exists $n_{0}$ such that $g^{n_{0}} f^{N+K l}(U) \geq 0$ which is inconsistent with equation (3).

2. There exists $n_{0}$ such that $\forall n \geq n_{0} \quad g^{n} f^{N+K l}(U) \widetilde{>} V$. In this case (see Fig. 3)

$$
\forall n \geq n_{0} \forall m \geq K \quad g^{n} f^{N+m l}(U) \geq g^{n} f^{N+K l}(U) \widetilde{>} V .
$$




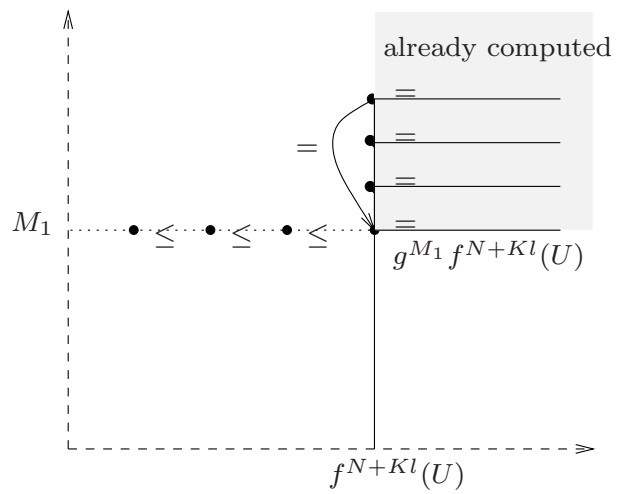

Figure 4. Case 3.

Thus we test if $V \in\left\{g^{n} f^{N+m l}(U) \mid n \in \mathbb{N}\right\}$ for all $m<K$ and we test if $V \in\left\{g^{n} f^{N+m l}(U) \mid m \in \mathbb{N}\right\}$ for all $n<n_{0}$ (which is decidable by Lem. 3.5 with $U^{\prime}=f^{N}(U)$ and $\left.f^{\prime}=f^{l}\right)$.

3. There exist $M_{1}, l_{1}$, such that (see Fig. 4)

$$
\forall n \geq M_{1} \quad \exists i<l_{1} \quad g^{n} f^{N+K l}(U)=g^{M_{1}+i} f^{N+K l}(U) \leq V .
$$

In this last case, we consider then:

$$
g^{M_{1}} f^{N+K l}(U) \geq g^{M_{1}} f^{N+(K-1) l}(U) \geq \cdots \geq g^{M_{1}} f^{N}(U)
$$

Applying Lemma 3.3 yields:

- either this sequence is already stabilized, so

$$
\forall m \geq K \quad g^{M_{1}} f^{N+m l}(U)=g^{M_{1}} f^{N+K l}(U)
$$

- or this sequence is $(k+1)$-almost increasing (at least until the $K^{\text {th }}$ term of the sequence), so:

$$
\begin{gathered}
\left|g^{M_{1}} f^{N+K l}(U)\right| \geq \frac{K}{k+1}+\left|g^{M_{1}} f^{N}(U)\right|=|V|+\left|g^{M_{1}} f^{N}(U)\right| \\
\Rightarrow g^{M_{1}} f^{N+K l}(U) \widetilde{>} V
\end{gathered}
$$

which is inconsistent with equation (5).

Thus we claim that

$$
\forall n \geq M_{1} \forall m \geq K \quad g^{n} f^{N+m l}(U)=g^{n} f^{N+K l}(U) .
$$


Indeed, let $n \geq M_{1}, m \geq K$,

$\left.g^{n} f^{N+m l}(U)=g^{n^{\prime}}\left(g^{M_{1}} f^{N+m l}(U)\right) \stackrel{(\mathrm{Eq} .}{=}(6)\right) g^{n^{\prime}}\left(g^{M_{1}} f^{N+K l}(U)\right)=g^{n} f^{N+K l}(U)$.

Thus $\left\{g^{n} f^{N+m l}(U) \mid n, m \in \mathbb{N}\right\}=\bigcup_{n \leq M_{1}}\left\{g^{n} f^{N+m l}(U) \mid m \in \mathbb{N}\right\}$

$$
\cup \bigcup_{m \leq K}\left\{g^{n} f^{N+m l}(U) \mid n \in \mathbb{N}\right\}
$$

Thus $V \in\left\{g^{n} f^{N+m l}(U) \mid n, m \in \mathbb{N}\right\}$ is decidable which completes the proof.

\section{UNDECIDABILITY IN THE GENERAL CASE}

In the general case: if an arbitrary number $p$ of functions are iterated in a fixed order, $V \in\left\{f_{p}^{n_{p}} \ldots f_{1}^{n_{1}}(U) \mid n_{1}, \ldots, n_{p} \in \mathbb{N}\right\}$ becomes undecidable.

Theorem 4.1. Given $f_{1}, \ldots, f_{p} \in \mathcal{A}_{k}(\mathbb{N}), U, V \in \mathbb{N}^{k}$, then $V \in\left\{f_{p}^{n_{p}} \cdots f_{1}^{n_{1}}(U) \mid\right.$ $\left.n_{1}, \ldots, n_{p} \in \mathbb{N}\right\}$ is undecidable.

We present here the sketch of the proof, lemmas needed for the proof are developed in Sections 4.1, 4.2 and 4.3, we conclude with the proof in Section 4.4. Section 4.5 presents a refinement of Theorem 4.1 .

Sketch proof. We start from Theorem 3.10 of [8] which is a stronger form of Hilbert's Tenth Problem, shown to be equivalent:

Theorem 4.2. [8] There is a polynomial $\mathcal{P}\left(x, y_{1}, \ldots, y_{N_{0}}\right)$ with integer coefficients such that no algorithm exists for deciding whether or not an arbitrary equation on the form

$$
\mathcal{P}\left(x_{0}, y_{1}, \ldots, y_{N_{0}}\right)=0
$$

where $x_{0}$ is a positive integer, has a solution in nonnegative integers $y_{1}, \ldots, y_{N_{0}}$.

From now on, $\mathcal{P}$ is reserved for the polynomial mentioned in Theorem 4.2 and $N_{0}$ is reserved for the degree of $\mathcal{P}$ minus 1 .

A straightforward corollary is the following:

Corollary 4.3. There is no algorithm for deciding whether or not an arbitrary equation on the form $Q\left(x_{1}, \ldots, x_{N_{0}}\right)=0$ where $Q$ is a polynomial with integer coefficients and $N_{0}$ variables, has a solution in nonnegative integers $x_{1}, \ldots, x_{N_{0}}$.

The idea of the proof is to establish a correspondence between each polynomial $P$ of $N_{0}$ variables and a system of affine functions (computable from $P$ ) which simulates the computation of $P\left(x_{1}, \ldots, x_{N_{0}}\right)$ for each tuple of $N_{0}$ integers.

We establish the correspondence in the following way:

(1) the first functions $C_{m_{j}, x_{i}}$ create a tuple of integers (see Lem. 4.4);

(2) the functions $f_{m_{j}, i}$ compute the monomials of $P$ (Lem. 4.5); 
(3) the functions $A_{m_{i}}^{+}, A_{m_{i}}^{-}, D$ compute $P$, keeping positiveness at each step (Lem. 4.7).

Then we conclude with Corollary 4.3.

Notation: If $m$ is the monomial $a_{\alpha_{1}, \ldots, \alpha_{N_{0}}} x_{1}^{\alpha_{1}} \ldots x_{N_{0}}^{\alpha_{N_{0}}}$, let $N_{m}$ denote $2\left(\alpha_{1}+\right.$ $\left.\cdots+\alpha_{N_{0}}\right)-1$ and $K_{m}=\frac{N_{m}-1}{2}$.

\subsection{Preparation Step: Generation of tuple of integers}

Lemma 4.4. For every monomial $m=a_{\alpha_{1}, \ldots, \alpha_{N_{0}}} x_{1}^{\alpha_{1}} \ldots x_{N_{0}}^{\alpha_{N_{0}}}$, there exist $N_{0}$ affine functions $C_{m, x_{1}}, \ldots, C_{m, x_{N_{0}}} \in \mathcal{A}_{N_{m}}(\mathbb{N})$ such that $\forall a_{1}, \ldots, a_{N_{0}} \in \mathbb{N}$,

$$
\left.C_{m, x_{N_{0}}}^{a_{N_{0}}} \cdots C_{m, x_{1}}^{a_{1}} O_{N_{m}}=\left(\begin{array}{c}
a_{1} \\
\vdots \\
a_{1} \\
\vdots \\
a_{N_{0}} \\
\vdots \\
a_{N_{0}} \\
0 \\
\vdots \\
0
\end{array}\right) \quad\right\} \alpha_{1}
$$

where $O_{N_{m}}$ is the null vector of size $N_{m}$. More formally, if $\alpha_{1}+\cdots+\alpha_{j} \leq i$ $<\alpha_{1}+\cdots+\alpha_{j}+\alpha_{j+1}$, the $i^{\text {th }}$ coordinate of $C_{m, x_{N_{0}}}^{a_{N_{0}}} \ldots C_{m . x_{1}}^{a_{1}} O_{N_{m}}$ is equal to $a_{j}$.

Proof of Lemma 4.4. We build $N_{0}$ functions $C_{m, x_{j}}=I d+V_{m, x_{j}}$ where the $i^{\text {th }}$ coordinate of $V_{m, x_{j}}$ is 1 if $\alpha_{1}+\cdots+\alpha_{j} \leq i<\alpha_{1}+\cdots+\alpha_{j}+\alpha_{j+1}, 0$ otherwise.

\subsection{Multiplications Functions}

\subsubsection{Definitions}

We generalize Example 1.1: if $i, j, l, N$ are distinct integers $(N \geq i, j, l)$, there exists a function $f_{i . j \rightarrow l}: \mathbb{N}^{N} \rightarrow \mathbb{N}^{N}$ such that $f_{i . j \rightarrow l}$ computes the product of the $i^{\text {th }}$ coordinate by the $j^{\text {th }}$ and writes the result on the $l^{\text {th }}$ coordinate:

$$
\exists n \quad f_{i . j \rightarrow l}^{n}\left(x_{i} E_{i}^{N}+x_{j} E_{j}^{N}\right)=x_{j} E_{j}^{N}+d E_{l}^{N} \text { iff } d=x_{i} x_{j} .
$$


4.2.2. Computation of the monomials

Lemma 4.5. For every monomial $m=a_{\alpha_{1}, \ldots, \alpha_{N_{0}}} x_{1}^{\alpha_{1}} \ldots x_{N_{0}}^{\alpha_{N_{0}}}$, there exist $K_{m}$ affine functions $f_{m, 1}, \ldots, f_{m, K_{m}} \in \mathcal{A}_{N_{m}}(\mathbb{N})$ such that $\forall a_{1}, \ldots, a_{K_{m}+1} \in \mathbb{N}$,

$$
\exists k_{1}, \ldots, k_{K_{m}} \quad f_{m, K_{m}}^{k_{K_{m}}} \cdots f_{m, 1}^{k_{1}}\left(\begin{array}{c}
a_{1} \\
\vdots \\
\vdots \\
a_{K_{m}+1}, \ldots, c_{K_{m}} \\
0 \\
\vdots \\
0
\end{array}\right)=\left(\begin{array}{c}
0 \\
c_{1} \\
0 \\
\vdots \\
0 \\
c_{2} \\
\vdots \\
c_{K_{m}} \\
b
\end{array}\right) \text { iff } b=a_{1} \cdots a_{K_{m}+1}
$$

Proof of Lemma 4.5. Let $f_{m, 1}=f_{1,2 \rightarrow K_{m}+2}, f_{m, i}=f_{i+1, K_{m}+i \rightarrow K_{m}+i+1}$ for $2 \leq i \leq K_{m}$

Together with Lemma 4.4, we obtain a straightforward corollary:

Corollary 4.6. For every monomial $m=a_{\alpha_{1}, \ldots, \alpha_{N_{0}}} x_{1}^{\alpha_{1}} \ldots x_{N_{0}}^{\alpha_{N_{0}}}$, there are affine functions $f_{m, 1}, \ldots, f_{m, K_{m}}, C_{m, x_{1}}, \ldots, C_{m, x_{N_{0}}} \in \mathcal{A}_{N_{m}}(\mathbb{N})$ such that

$$
\begin{aligned}
& \left.\left\{b \in \mathbb{N} \mid \begin{array}{l}
\exists k_{1}, \cdots, k_{K_{m}} \in \mathbb{N}, \\
\exists c_{1}, \ldots, c_{K_{m}} \in \mathbb{N}, \quad f_{m, K_{m}}^{k_{K_{m}}} \cdots f_{m, 1}^{k_{1}} C_{m, x_{N_{0}}}^{a_{N_{0}}} \cdots C_{m, x_{1}}^{a_{1}} O_{N_{m}}= \\
\exists a_{1}, \ldots, a_{N_{0}} \in \mathbb{N}, \\
c^{\prime} \\
\vdots \\
c_{K_{m}} \\
b
\end{array}\right)\right\} \\
& =\left\{n_{1}^{\alpha_{1}} \cdots n_{N_{0}}^{\alpha_{N_{0}}} \mid n_{1}, \ldots, n_{N_{0}} \in \mathbb{N}\right\} .
\end{aligned}
$$

4.3. Computation of $P$

Lemma 4.7. Let $P$ be a polynomial of $N_{0}$ variables, whose constant term is 0 . There exists $k$, there exist affine functions $f_{1}, \ldots, f_{n} \in \mathcal{A}_{k}(\mathbb{N})$ such that

$\left\{b \in \mathbb{N} \mid \exists k_{1}, \cdots k_{n} \quad f_{1}^{k_{1}} \ldots f_{n}^{k_{n}} 0_{k}=\left(\begin{array}{c}0 \\ \vdots \\ 0 \\ b\end{array}\right)\right\}=\left\{P\left(n_{1}, \cdots, n_{N_{0}}\right) \mid n_{1}, \ldots, n_{N_{0}} \in \mathbb{N}\right\}$

Proof of Lemma 4.7. Let $P$ be a polynomial of $N_{0}$ variables, whose constant term is 0 . 
For each monomial $m$ of $P$, we construct affine functions

$$
f_{m, 1}, \ldots, f_{m, K_{m}}, C_{m, x_{1}}, \ldots, C_{m, x_{N_{0}}} \in \mathcal{A}_{N_{m}}(\mathbb{N})
$$

as described in Corollary 4.6.

We establish a one-to-one correspondence between each monomial $m$ of $P$ and a "block" $\mathcal{B}_{m}$ whose size is $N_{m} \times N_{m}$. From now on, we will only consider block matrix and block vectors, of the form:

$$
\left(\begin{array}{cccc|c}
\mathcal{B}_{m_{1}} & 0 & 0 & 0 & 0 \\
0 & \mathcal{B}_{m_{2}} & 0 & 0 & \vdots \\
0 & 0 & \ddots & 0 & \vdots \\
0 & 0 & 0 & \mathcal{B}_{m_{L}} & 0 \\
\hline 0 & \cdots & \cdots & 0 & 1
\end{array}\right) \quad, \quad\left(\begin{array}{c}
\mathcal{V}_{m_{1}} \\
\mathcal{V}_{m_{2}} \\
\vdots \\
\mathcal{V}_{m_{L}} \\
a
\end{array}\right)
$$

where $L$ is the number of monomials.

We transform the functions $f_{m, i}$ and $C_{m, x_{i}}$ into functions $\tilde{f}_{m, i}$ and $\tilde{C}_{m, x_{i}}$ in $\mathcal{A}_{N+1}(\mathbb{N})$, where $N=\sum_{i=1}^{K} N_{m_{i}}$, in the following way:

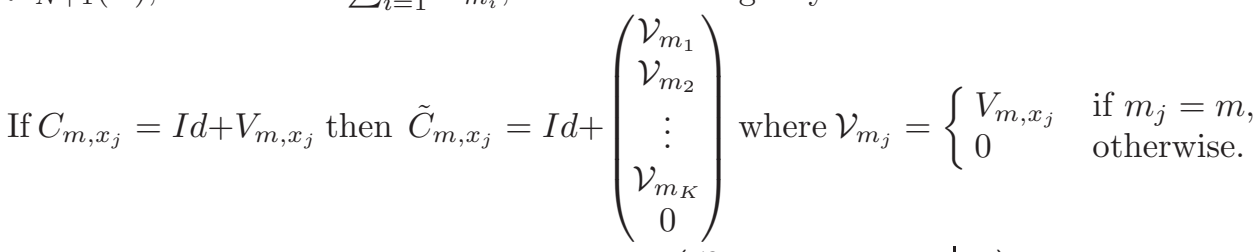

If $f_{m, i}(X)=A_{m, i} X+V_{m, i}$, then $\tilde{f}_{m, i}(X)=\left(\begin{array}{cccc|c}\mathcal{B}_{m_{1}} & 0 & 0 & 0 & 0 \\ 0 & \mathcal{B}_{m_{2}} & 0 & 0 & \vdots \\ 0 & 0 & \ddots & 0 & \vdots \\ 0 & 0 & 0 & \mathcal{B}_{m_{K}} & 0 \\ \hline 0 & \cdots & \cdots & 0 & 1\end{array}\right) X+\left(\begin{array}{c}\mathcal{V}_{m_{1}} \\ \mathcal{V}_{m_{2}} \\ \vdots \\ \mathcal{V}_{m_{K}} \\ 0\end{array}\right)$

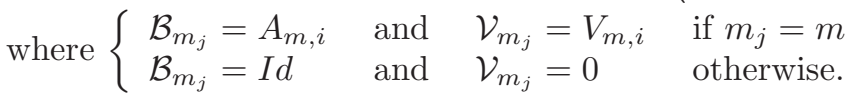

Besides, for each $m$, let

$$
I_{m}=\left\{i_{m}, i_{m}+2, i_{m}+3, \ldots, i_{m}+K_{m}-1, i_{m}+K_{m}, i_{m}+N_{m}-1\right\}
$$

where $i_{m}$ is the coordinate of the first line of block $\mathcal{B}_{m}$. This set corresponds to the coordinates we have to test: after the iterations of $f_{m, 1}$, then $f_{m, 2}$, then, ... then $f_{m, K_{m}}$, we obtain the product of the $K_{m}+1$ first coordinates if and only if the coordinates whose numbers are in $I_{m}-\left\{i_{m}+N_{m}-1\right\}$ are equal to zero.

We split the monomials of $P$ into 2 categories:

$$
I=\left\{m \mid a_{\alpha_{1}, \ldots, \alpha_{N_{0}}} \geq 0\right\}, \quad J=\left\{m \mid a_{\alpha_{1}, \ldots, \alpha_{N_{0}}}<0\right\} .
$$

$\left(a_{\alpha_{1}, \ldots, \alpha_{N_{0}}}\right.$ is the coefficient of the monomial $\left.m=a_{\alpha_{1}, \ldots, \alpha_{N_{0}}} x_{1} \ldots x_{N_{0}}.\right)$ 
For every $m$ in $I$ (resp. in $J$ ), we define:

$$
A_{m}^{+}\left(\text {resp. } A_{m}^{-}\right)=I d-E_{i_{m}+2 K_{m}}^{N+1}+a_{\alpha_{1}, \ldots, \alpha_{N_{0}}} E_{N+1}^{N+1} .
$$

$i_{m}+2 K_{m}$ is the number of the line where the multiplication's result of $x_{1}^{n_{1}} \cdots x_{N_{0}}^{n_{N_{0}}}$ is written.

The aim of this distinction between $m \in I$ and $m \in J$ is to iterate first all the $A_{m}^{+}$ (we first add the nonnegative terms), then the $A_{m}^{-}$. In this way, if $P\left(a_{1}, \ldots, a_{N_{0}}\right)$ $\geq 0$, we make sure that during the computation of $P\left(a_{1}, \ldots, a_{N_{0}}\right)$, the intermediate vectors remain nonnegative at each iteration.

Let $D$ denote a diagonal matrix such that if $i \in \bigcup_{m} I_{m}$ then $D_{i, i}=1$ else $D_{i, i}=0$. Actually, $D$ assigns irrelevant coordinates to 0 .

Applying Corollary 4.6, we obtain the following equivalence:

$$
\exists x_{1} \ldots \exists x_{N_{0}} \quad P\left(x_{1}, \ldots, x_{N_{0}}\right)=a \quad a \geq 0
$$

if and only if

$$
\begin{gathered}
\exists n_{D} \exists n_{A_{m_{1}}}^{-} \ldots \exists n_{A_{m_{M}}}^{-} \exists n_{A_{m_{1}}}^{+} \ldots \exists n_{A_{m_{M}}}^{+} \\
\exists n_{m_{1}, 1} \ldots \exists n_{m_{1}, K_{m_{1}}} \ldots \exists n_{m_{L}, 1} \ldots \exists n_{m_{L}, K_{m_{L}}} \exists n_{1} \ldots \exists n_{N_{0}} \\
D^{n_{D}}\left(\prod_{i=1}^{M} A_{m_{1}}^{-} n_{A_{m_{1}}}^{-}\right)\left(\prod_{i=1}^{M^{\prime}} A_{m_{1}}^{+} n_{A_{m_{1}}}^{+}\right)\left(\prod_{i=1}^{L} \prod_{j=1}^{K_{m_{i}}} f_{m_{i}, j}^{n_{m_{i}, j}}\right)\left(\prod_{i=1}^{N_{0}} C_{x_{i}}^{n_{i}}\right)\left(\begin{array}{c}
0 \\
\vdots \\
0 \\
0
\end{array}\right)=\left(\begin{array}{c}
0 \\
\vdots \\
0 \\
a
\end{array}\right)
\end{gathered}
$$

where $L$ is the number of monomials, $M$ the number of nonnegative monomials and $M^{\prime}$ the number of negative monomials. This completes the proof of Lemma 4.7. $\square$

\subsection{Conclusion: Reduction to Hilbert's tenth Problem}

We can now prove Theorem 4.1.

Proof. For each polynomial $P$ of degree $N_{0}$, we associate:

$$
\tilde{P}= \begin{cases}P-a_{0, \ldots, 0} & \text { if } a_{0, \ldots, 0}<0 \\ -P+a_{0, \ldots, 0} & \text { if } a_{0, \ldots, 0} \geq 0\end{cases}
$$

Then $P\left(x_{1}, \ldots, x_{N_{0}}\right)=0$ iff $\tilde{P}\left(x_{1}, \ldots, x_{N_{0}}\right)=\left|a_{0, \ldots, 0}\right|$.

The constant term of $\tilde{P}$ is 0 , thus we build

$$
D, A_{m_{1}}^{-}, \ldots, A_{m_{M}}^{-} A_{m_{1}}^{+}, \cdots, A_{m_{M^{\prime}}}^{+}, f_{m_{1}, 1}, \ldots, f_{m_{1}, N_{m_{1}}}, \ldots, f_{m_{L}, 1}, \ldots, f_{m_{L}, N_{m_{L}}}
$$

from $\tilde{P}$ as described in Section 4.3. 
Thus,

$$
\exists x_{1} \ldots \exists x_{N_{0}} \quad P\left(x_{1}, \ldots, x_{N_{0}}\right)=0
$$

if and only if

$$
\exists x_{1} \ldots \exists x_{N_{0}} \quad \tilde{P}\left(x_{1}, \ldots, x_{N_{0}}\right)=\left|a_{0, \ldots, 0}\right|
$$

if and only if (see Sect. 4.3)

$$
\left(\begin{array}{c}
0 \\
\vdots \\
0 \\
\left|a_{0, \ldots, 0}\right|
\end{array}\right) \in\left\{D^{n_{D}} \ldots C_{x_{N_{0}}}^{n_{N_{0}}}\left(\begin{array}{c}
0 \\
\vdots \\
0 \\
0
\end{array}\right) \mid n_{D}, \ldots, n_{N_{0}} \in \mathbb{N}\right\}
$$

If $V \in\left\{f_{p}^{n_{p}} \ldots f_{1}^{n_{1}}(U) \mid n_{1}, \ldots, n_{p} \in \mathbb{N}\right\}$ was decidable, then the Hilbert's tenth problem would be decidable too.

Conclusion: $V \in\left\{f_{p}^{n_{p}} \cdots f_{1}^{n_{1}}(U) \mid n_{1}, \ldots, n_{p} \in \mathbb{N}\right\}$ is undecidable.

\subsection{EXISTENCE OF A BOUND}

We have shown that, given $p \in \mathbb{N}, f_{1}, \ldots, f_{p} \in \mathcal{A}_{k}(\mathbb{N}) U, V \in \mathbb{N}^{k}$, the problem $V \in\left\{f_{p}^{n_{p}} \cdots f_{1}^{n_{1}}(U) \mid n_{1}, \ldots, n_{p} \in \mathbb{N}\right\}$ is undecidable.

Actually, the number of functions $f_{i}$ can be fixed in advance (provided the number of functions is large enough), it is not a parameter of the problem:

Theorem 4.8. There are $f_{1}, \ldots, f_{p}$ affine functions in $\mathcal{A}_{k}(\mathbb{N})$ such that, given $U, V \in \mathbb{N}^{k}$, the problem $V \in\left\{f_{p}^{n_{p}} \cdots f_{1}^{n_{1}}(U) \mid n_{1}, \ldots, n_{p} \in \mathbb{N}\right\}$ is undecidable.

It follows that:

Corollary 4.9. There exists $K \in \mathbb{N}$, such that: for all " $i x e d " ~ p \geq K$, given $f_{1}, \ldots, f_{p} \in \mathcal{A}_{k}(\mathbb{N}), U, V \in \mathbb{N}^{k}$, the problem $V \in\left\{f_{p}^{n_{p}} \cdots f_{1}^{n_{1}}(U) \mid n_{1}, \ldots, n_{p} \in \mathbb{N}\right\}$ is undecidable.

Proof. To show this last result, we re-use the strong form of Hilbert's tenth problem: Theorem 3.10 of [8], cited here as Theorem 4.2.

We associate with the polynomial $\mathcal{P}$ (defined in Th. 4.2), affine functions $C_{x}$, $C_{y_{i}}, f_{m_{i}, j}, A_{m_{i}}^{+}, A_{m_{i}}^{-}, D \in \mathcal{A}_{N+1}(\mathbb{N})$ as in Section 4.4. Let $a$ be the constant term of $\mathcal{P}$. Then: given $n_{0}, \mathcal{P}\left(n_{0}, y_{1}, \ldots, y_{m}\right)=0$ has a solution in nonnegative integers $y_{1}, \ldots, y_{m}$ iff

$$
V \in\left\{D^{n_{D}} \cdots C_{y_{1}}^{n_{1}} \cdots C_{y_{m}}^{n_{m}} U \mid n_{D}, \ldots, n_{1}, \ldots, n_{m} \in \mathbb{N}\right\}
$$

where $U=C_{x}^{n_{0}} O_{N+1}$ and $V=|a| E_{N+1}^{N+1}$. This is not decidable, so we complete the proof. 


\section{Conclusion}

We have proved that the original reachability problem is undecidable for some fixed number $p$ of functions and that it is decidable for $p=1$ and $p=2$. There are some restrictions on the $f_{i}$ which restore the decidability: for example, if $\forall i \forall X \quad\left|f_{i}(X)\right| \geq X$ (where $|V|$ is the sum of the absolute values of its coordinates) or if each $B_{i}$ is nonnegative. These minor results are not shown here (see [4]).

Acknowledgements. I would like to thank the anonymous referees for their helpful and precise suggestions and comments.

\section{REFERENCES}

[1] E. Asarin, G. Schneider and S. Yovine, Towards computing phase portraits of polygonal differential inclusions, in HSCC'2002, Hybrid Systems: Computation and Control. Stanford, USA, Lecture Notes in Comput. Sci. 2289 (2002) 49-61.

[2] B. Boigelot, Symbolic Methods for Exploring Infinite Sate Spaces, Ph.D. Thesis. Université de Liège (1998) 225.

[3] H. Comon and Y. Jurski, Multiple counters automata, safety analysis and Presburger arithmetic, in Proc. Computer Aided Verification. Springer Verlag, Lecture Notes in Comput. Sci. 1427 (1998) 268-279.

[4] V. Cortier, Vérification de systèmes à compteurs (in French), Master's Thesis. Université Paris 7 (1999) http://www.lsv.ens-cachan.fr/ cortier/memoire_dea.ps

[5] L.E. Dickson, Finiteness of the odd perfect and primitive abundant numbers with $n$ distinct prime factors. Amer. J. Math. 35 (1913) 413-422.

[6] C. Dufourd, A. Finkel and Ph. Schnoebelen, Between decidability and undecidability, in Proc. ICALP 1998. Springer-Verlag, Lecture Notes in Comput. Sci. 1448 (1998) 103-115.

[7] A. Finkel, P. McKenzie and C. Picaronny, A well-structured framework for analysing Petri nets extensions. Technical Report, Research Report LSV-99-2 (1999) http://www.lsv.ens-cachan.fr/Publis/RAPPORTS_LSV/rr-lsv-1999-2.rr.ps

[8] M.Yu. Matijacevitch, M. David and J. Robinson, Hilbert's Tenth Problem, Chapter 3 (1976).

[9] C. Rorres and H. Anton, Applications of Linear Algebra, Chapters 9 and 13 (1979).

Communicated by S. Tison.

Received May, 2001. Accepted October, 2002.

To access this journal online: www.edpsciences.org 